\title{
El derecho a una vida libre de algoritmos*
}

\section{Guillermo Antonio Tenorio Cueto**}

\begin{abstract}
RESUMEN
El presente trabajo tiene como objetivo central plantar la posibilidad del surgimiento de un derecho humano derivado de la autodeterminación informativa e inmerso en la vida privada de las personas que pretenda excluir a las mismas de la obligación de participar en procesos tecnológicos donde su vida privada, su vida intima, su propia imagen y sus datos personales sean comprometidos, analizados, explorados y abusados por organizaciones públicas y privadas. El eje central del trabajo estará vinculado a la predicción y segmentación psicológica, de comportamientos e intereses, asi como la segmentación algorítmica con finalidades de perfilamiento para diversos fines comerciales, politicos, de vigilancia, entre otros, de igual manera a la analítica prescriptiva tendiente a la eliminación de participación humana en la toma de decisiones. Se propone, luego de revisar los alcances y los impactos de las nuevas tecnologías de inteligencia artificial y machine learning, explorar los alcances $y$ efectos del derecho proponiendo la garantía del Estado de vigilar y evitar efectos discriminatorios por la negativa del consentimiento de participar en estos procesos vinculados a cualquier tratamiento de datos
\end{abstract}

\section{PALABRAS CLAVE}

Derecho a la información; derecho a la privacidad, algoritmos, análisis predictivo.

\begin{abstract}
The paper has as a central objective to plant the possibility of the emergence of a human right derived from the informative self-determination and inmersed in the private life of people who intend to exclude them from the obligation to participate in technological processes where their private life, their intimate life, your own image and your personal data are compromised, analyzed, explored and abused by public and private organizations. The central axis of this paper will be linked to the prediction and psychological segmentation of behaviors and interests, as well as the algorithmic segmentation with porpuse of profiling for varios comercial, political, surveillance, among others and in the same way to the prescriptive analytical tending to the elimination of human participation in decision making. It is proposed, after reviewing the scope and impacts of the new artificial intelligence and machine learning tecnologies, to explore the scope and effects of the law, proposing the State's guarantee to monitor and avoid discriminatory effects due to the refusal of consent to participate in these processes linked to any data processing.
\end{abstract}

\section{KEYWORDS}

Rigth to information, right to privacy; Algorithms, predictive analysis.

\footnotetext{
*Artículo de Investigación postulado el 21 de febrero de 2020 y aceptado el 30 de septiembre de 2020

**Profesor investigador en la Universidad Panamericana, Ciudad de México. (gtenorio@up.edu.mx) orcid.org/00000003-1719-2216
} 


\section{SUMARIO}

1. Nota introductoria

2. Los efectos de la tecnología en los derechos humanos

3. La segmentación algorítmica y su impacto en la vida privada

a) Breve excursus sobre la vida privada

b)Segmentación algoritmica, análisis predictivo y machine learning observando y aprendiendo de nuestra vida privada

4. ¿Es posible una vida libre de algoritmos, segmentación o análisis predictivo?

5. Conclusiones

6. Bibliografía.

\section{Nota introductoria.}

La profunda disyuntiva que plantea el título del presente trabajo transporta al lector sólo a dos caminos posibles. Por un lado, suponer que los nuevos desarrollos tecnológicos son el único camino posible de evolución, comodidad o seguridad y que garantizan la potencialidad de diversos derechos humanos que al parecer sólo se alcanzan de manera plena con el mencionado desarrollo. Por el otro, suponer que los mismos desarrollos impactan de manera tan negativa en la esencia misma del ser humano como en su intimidad, en su libertad o en la igualdad, nos conduce a pensar el replanteamiento acerca de nuestra relación con la tecnología y sobre todo a ser cautelosos al extremo respecto a su impacto en nuestra vida cotidiana.

Adicionalmente a lo anterior es pertinente señalar que el título del trabajo es sugerente al emplear la palabra "algoritmo" como un recurso de impacto que de alguna manera recoge lo que en el lenguaje cotidiano suponen las personas que realizan todas las propuestas tecnológicas contemporáneas englobando inteligencia artificial, máquinas de aprendizaje, robótica o la misma segmentación algorítmica. Es importante precisar que el lector podrá encontrar en el trabajo una diferenciación entre todas las propuestas tecnológicas.

Hablar de una vida fuera del ámbito de la tecnología sería como proponer una vida en "la caverna" haciendo alusión al famoso mito de Platón. Los desarrollos tecnológicos han acompañado al ser humano desde siempre y al menos desde la Revolución Industrial han planteado una sustancial modificación del comportamiento de nuestra vida en sociedad. Pensar hoy en una vida sin teléfono, sin autos, sin computadoras o sin aviones sería prácticamente impensable. En ese sentido el trabajo no propondrá una ruptura con la tecnología sino sólo planteará la pregunta sobre si toda la tecnología supone progreso alineado 
al respeto de la dignidad del Ser Humano o no y si no lo hace, nos preguntaremos si es factible la elección de decidir no participar en dicho proceso tecnológico con la debida protección y garantía del Estado de evitar su obligatoriedad.

El trabajo se ha estructurado de la siguiente manera con la cual creemos que podemos llegar a una aproximación para responder la pregunta que se formuló en el párrafo anterior. En un primer momento trabajaremos de manera genérica sobre la relación entre tecnología y derechos humanos haciendo énfasis particular en los casos donde dichos derechos son violentados por los efectos de la aplicación tecnológica. Evidenciaremos sus consecuencias. La segunda parte del trabajo lo dedicaremos a la vida privada e íntima, pues más allá de otras repercusiones, es en los derechos humanos donde pretendemos radicar la evidencia de la violación extrema de las nuevas tecnologías y en donde encontraremos los efectos más violentos respecto a la dignidad humana. Ahí abordaremos las posiciones transhumanistas que conllevan necesariamente la reducción de la persona a un conjunto de procesos bioquímicos nulificando su capacidad electiva. En un tercer momento propondremos una alternativa que condicione al Estado a proteger y garantizar un derecho vinculado con la libertad del Ser Humano de participar o no en estos procesos de nueva tecnología.

\section{Los efectos de la tecnología en los derechos humanos}

Durante el año 2014 Canadá tomó la decisión de incorporar un sistema de aprendizaje algorítmico automatizado para lograr procesar un número elevado de peticiones sobre migración que recibían las oficinas que atendían estos casos. Desde luego la cantidad, no sólo de solicitudes sino de datos que se vinculaban en su conjunto a las mismas era enorme, ocasionando que cada procesamiento fuera muy lento y representara muchísimo trabajo para sus oficinas. La adquisición de este sistema automatizado le permitiría a Canadá poder resolver de manera eficiente la gestión de dichas solicitudes. Unos años más tarde, en el 2018 se detectó un serio problema sobre el aprendizaje automatizado del sistema que podría tener consecuencias lamentables en torno a las decisiones que tomaba la máquina respecto a las solicitudes, pues colocaba a las personas altamente vulnerables en situaciones de procesos injustos e ilegales. ${ }^{1}$

La referencia al caso anteriormente descrito, evidencia la profunda e intima relación existente entre los dos conceptos que proponemos para este

\footnotetext{
${ }^{1}$ Cfr. Molnar, Petra, LEX, Gill, Bots at the gate: A human rights analysis of automated decisión - making in Canada s inmigration and refugee system, Citizen Lab University of Toronto, 2018. [Consultado el 10 de febrero de 2020] Disponible en: https://citizenlab.ca/wp-content/uploads/2018/09/IHRP-Automated-Systems-Report-Web-V2.pdf
} 
apartado. Los llamados derechos humanos en relación con la tecnología deben aparecer de manera vigorosa para exigir que el uso de la nueva tecnología, no participe en procesos de vulneración de aquellos. En ese sentido se actualiza toda la tradición que en materia de derechos humanos se ha acuñado respecto a su impacto, radicalidad, aparición y consecuencias. Así, Antonio E. Pérez Luño afirma que:

(...) los derechos humanos son un conjunto de facultades e instituciones que en cada momento histórico concretan las exigencias de la dignidad, la libertad y la igualdad humanas, las cuales deben ser reconocidas positivamente por los ordenamientos jurídicos a nivel nacional e internacional. $^{2}$

En ese tenor los derechos humanos no se encuentran encapsulados en un tiempo determinado, por el contrario, el devenir histórico obliga al Estado a su reconocimiento, actualización y defensa permanente en aras no sólo de la protección individual sino del sano desarrollo de la comunidad. En ese sentido, José Castán Tobeñas nos refiere que los derechos del hombre "deben ser reconocidos y respetados por todo poder y norma jurídica positiva, cediendo no obstante en su ejercicio ante las exigencias del bien común". ${ }^{3}$ A la par es imperante referir que dichos derechos humanos no son una graciosa concesión del Estado sino por el contrario, éste se encuentra obligado a su reconocimiento en virtud de que aquellos se muestran de manera intrínseca en la naturaleza misma de la persona, en su dignidad específica. Esto fraseado de otra manera significa que por el simple y sencillo hecho de ser personas gozamos a plenitud de ellos siendo obligatorio su respeto y cuidado. Sobre el particular existen diversos autores que así lo refieren y que para no distraer la lectura de nuestro tema principal sólo los mostraremos como referencia. ${ }^{4}$

Ante ello es inevitable afirmar que el respeto a los derechos humanos supone un ejercicio colectivo, es decir, todos estamos llamados a protegerlos, res118 petarlos y cuidarlos mientras que el Estado estará llamado a garantizar dicha protección poniendo a disposición de cada persona en lo individual todos y

\footnotetext{
${ }^{2}$ Cfr. Pérez Luño, Antonio E, Los derechos fundamentales, Madrid, Tecnos, 2004, p. 18

${ }^{3}$ Cfr. Castán Tobeñas, José, Los derechos del hombre, Madrid, Reus, 1992, p. 35

${ }^{4}$ De manera enunciativa se refieren Ramirez García, H. Pallares Yabur, P. Derechos humanos, Oxford, México, 2011. Carbonell, M., El ABC de los derechos humanos y el control de convencionalidad, México, Porrúa, 2014. Morales Vega, L., Derechos humanos y su interpretación en la Corte, México, Dofiscal, 2018. Beitz, Ch., La idea de derechos humanos, España, Marcial Pons, 2014. Hierro, L., Los derechos humanos, una concepción de la justicia, España, Marcial Pons, 2016.
} 
cada uno de los mecanismos necesarios para conseguir dicha protección. En ese sentido en prácticamente todos los sistemas de corte democrático encontraremos instituciones, recursos y procesos vinculados a la protección de los derechos humanos. Por si fuera poco, en el ámbito internacional encontramos convenciones internacionales que amplían su contenido, que promueven principios y que contribuyen no sólo a su protección sino a la debida diligencia que todos los Estados deben poner para su resguardo.

De todo lo anterior desprendemos que los desarrollos tecnológicos no se escapan de ello. De hecho, la tecnología está llamada a colaborar con la concreción de este ideario de los derechos humanos. Baste observar cómo al menos en el caso del derecho a la información, los desarrollos tecnológicos han impactado favorablemente a las personas. El tener acceso a la información de naturaleza pública como pueden ser el conocimiento en sí, servicios, trámites o productos que impactan en el desarrollo de las comunidades significa un determinante progreso para las mismas. Así el productor del campo puede conocer mejores técnicas de cultivo o producción, puede llevar a cabo trámites, pagar o contratar servicios o vender su producto desde el lugar donde vive sin menoscabar tiempo ni dinero en movilidad tramitológica. Esto se ha conocido nada más y nada menos como sociedad de la información la cual "es un nuevo modelo de organización industrial, cultural y social caracterizado por el acercamiento de las personas a la información a través de las nuevas tecnologías de la comunicación". ${ }^{5}$ Desde luego que dicho cambio de paradigma sin la presencia tecnológica hubiera sido impensable.

Sin embargo, conforme los desarrollos tecnológicos plantean mayores y más acelerados retos de manera concomitante aparecen situaciones de riesgo, vulneración o menoscabo de los derechos de las personas y es ahí, donde es necesario advertir los peligros que suponen dichos desarrollos para todos. Así por ejemplo, las cámaras de video vigilancia con motivo de la seguridad (tema al que volveremos más adelante) se justifican a plenitud cuando se argumenta a su favor un mejor y mayor control de la prevención del crimen en las calles de las ciudades pero que desde otra perspectiva, alimentan el temor de las personas cuando sabemos que, sobre la información recabada respecto a nuestro comportamiento en la vía pública, es utilizada para fines diversos incluidos en algunos casos para proveer de información a la delincuencia organizada a partir de la colusión entre ésta y las autoridades encargadas de administrar dicha información. En ese mismo tenor y con la puesta en marcha de la tecnología de reconocimiento facial el

${ }^{5}$ Cfr. Campuzano Tome, Herminia, Vida Privada y Datos Personales, Madrid, Tecnos 2000 
temor se agrava cuando se establece un mecanismo de crédito social, de monitoreo constante o simplemente de patrones de movilidad que sólo corresponden a las personas. En ese sentido refiere Isabel Lucena-Cid que:

(...) la amenaza a los derechos fundamentales es evidente si no se establecen mecanismos de protección de los ciudadanos. Esto es especialmente cierto en el caso de personas y miembros de algunas minorías en las grandes ciudades, que ya de por sí pueden ser objeto de vigilancia e injustamente perseguidos por la policía y las autoridades locales". ${ }^{6}$

A la par Howard Rheingold ha sido enfático en señalar que "la vigilancia sobre millones de personas que están interactuando en línea debería preocuparnos tanto como cualquier otro tipo de vigilancia o control que podría llegar a ejercer sobre nosotros el Estado y otras entidades que operan en Internet". "

Como podemos apreciar la tecnología tiene un insumo fundamental que es la información. En ese sentido uno de los problemas medulares respecto al impacto en los derechos humanos por parte de aquella se encontrará inherente a la captación, uso, procesamiento y toma de decisiones que conlleve la misma. Así, en los dos casos relatados anteriormente (migración en Canadá y cámaras de seguridad) presentan el mismo problema que es un uso indiscriminado de la información en alguna de sus etapas que acaba terminando en una violación a los derechos humanos de una persona, un grupo o la sociedad entera. ${ }^{8}$

A pesar de ello la necesidad de una información abierta en todos los sentidos es algo que ha venido alimentando a la industria tecnológica desde hace varios años. La maximización de la libre información permite que redes sociales como Facebook, Instagram, Twitter o Youtube partan de una idea de la información libre donde la vida privada no es oponible al considerarse como plataformas de interacción social a partir de las vivencias personales. Y lo mismo pasa con Wikileaks, Google o Cambridge Analytica donde el insumo que genera las mayores cantidades de dinero son los datos y la información cruza120 da que de esos datos emigran. ¿Qué más da invadir la vida privada de las personas si podemos entender su comportamiento frente a determinadas marcas comerciales o preferencias políticas?

\footnotetext{
${ }^{6} \mathrm{Cfr}$. Lucena-Cid, Isabel, El impacto de las nuevas tecnologías en los derechos humanos, In: Revista Aporia Jurídica (on-line). Revista Juridica do Curso de Direito da Faculdade CESCAGE. 8a. Edição. Vol. 1 (jul/dez-2017). p.16 ${ }^{7}$ Rheingold, Howard, Multitudes inteligentes. La próxima revolución social, Barcelona: Gedisa, 2004, P. 35 ${ }^{8}$ Stallman, Richard, A radical proposal to keep your personal data safe, The Guardián, Abril 2018. Disponible en: https://www.theguardian.com/commentisfree/2018/apr/03/facebook-abusing-data-law-privacy-big-tech-surveillance, Fecha de última consulta el 6 de febrero de 2020.
} 
El principal cambio de paradigma que incide directamente lo describe a la perfección Yuval Harari al referir que:

La libertad de expresión se concedió a los humanos... La libertad de información, en cambio, no se concede a los humanos. Se concede a la información... este valor nuevo puede afectar a la tradicional libertad de expresión, al dar trato de favor al derecho de información para que circule libremente sobre el derecho de los humanos a poseer datos y a restringir su movimiento. ${ }^{9}$

Esta forma de entender la información y los datos que la componen impactarán en los siguientes años de manera determinante en múltiples derechos humanos que durante los últimos dos siglos se ha buscado proteger. La nueva forma de entender la relación con la tecnología aparenta implícitamente un menoscabo de la libertad, la privacidad, la intimidad, los derechos a un debido proceso o la igualdad como baluarte de la democracia contemporánea. La liberación de información es fundamentalmente liberación de datos ${ }^{10} \mathrm{en}$ donde la renuncia voluntaria a la privacidad y a otros derechos supone que los algoritmos conozcan mis gustos, aficiones, preferencias o lugares a donde me dirijo ${ }^{11}$ en aras de una mayor comodidad, seguridad e inmediatez.

Pero el desafío más radical para la defensa de los derechos humanos viene alrededor de una fuente tecnológica que, fuera de la mente y comportamiento propiamente humano, genera decisiones que afectan a las personas en términos de violación a su privacidad o en términos de discriminación. El arribo del llamado internet de las cosas, la segmentación algorítmica o el machine learning que han supuesto un nivel avanzado en términos de comodidad, conocimiento y procesamiento de grandes cantidades de datos hoy representan una frontera cada vez más lejana de los derechos humanos. Veamos a continuación los impactos y alcances que esto propicia.

\footnotetext{
${ }^{9}$ Cfr. Harari, Yuval, Homo Deus, Debate, México, 2016, p.416.

${ }^{10}$ Ibidem, p. 417.

${ }^{11}$ Ibidem, p. 418
} 


\section{La segmentación algorítmica y su impacto en la vida privada.}

\section{a) Breve excursus sobre la vida privada}

La llamada vida privada ha estado presente en toda la historia de la humanidad aún y cuando no ha sido objeto de estudio en toda ella ${ }^{12}$. Resulta curioso que las referencias que vamos encontrando invariablemente permanecen asociadas a la cara opuesta de la vida pública. En ese sentido algunas referencias de la misma la señalan en tanto que no es vida pública o en tanto que esta última es sucedánea de la primera ${ }^{13}$ reduciéndola a un ambiente oscuro o en sombras ${ }^{14}$ impidiendo observar a su interior para conceptualizarla o entender el alcance de su significado.

La palabra "privado" va transformándose como la vida pública también lo hace. La aparición del Estado Moderno como fenómeno político totalizante absorbe y dota de un nuevo contenido a estas palabras. "Privado" significará "... sin oficio público, sin ocupar cargo público o posición oficial, sin empleo que lo implique en los asuntos públicos. El público es, en contraposición a la privacidad, el poder público". ${ }^{15}$ Ello adquiere una relevancia importante pues evidencia prima facie porque los llamados servidores públicos siempre serán nuestra primera categoría en donde, respecto a sus actividades, el derecho a la vida privada prácticamente desaparece.

$\mathrm{Al}$ parecer estos primeros tratamientos de la llamada vida privada se encuentran claramente vinculados a las actividades o actos que en la cotidianidad se desarrollan pero en realidad, aquella "...se desenvuelve en infinitas gradaciones y matices que oscilan entre los polos de la absoluta publicidad - cuando la persona desaparece por completo bajo la vestidura social- y la absoluta soledad, en donde la persona vive integra y absolutamente su vida auténtica", ${ }^{16}$ teniendo inclusive que este llamado "polo de absoluta soledad" se desdobla o nos muestra una faceta más profunda de la vida privada que es la denominada "intimidad".

\footnotetext{
${ }^{12} \mathrm{Cfr}$. Suárez Crothers, Christian. El concepto de derecho a la vida privada en el derecho anglosajón y europeo. Rev. derecho (Valdivia). [online]. dic. 2000, vol.11 [citado 24 Noviembre 2015], p. 103-120. Disponible en la World Wide Web: $<$ http://mingaonline.uach.cl/scielo.php?script=sci_arttextEtpid=S071809502000000100010\&tng=es\&tnrm=iso>

${ }^{13} \mathrm{Cfr}$. Arendt Hannah, La condición humana, PAIDOS, Barcelona, 2005

${ }^{14} \mathrm{El} \mathrm{juego} \mathrm{entre} \mathrm{la} \mathrm{luz} \mathrm{de} \mathrm{la} \mathrm{publicidad} \mathrm{y} \mathrm{la} \mathrm{oscuridad} \mathrm{de} \mathrm{la} \mathrm{vida} \mathrm{privada} \mathrm{lo} \mathrm{manifiesta} \mathrm{Habermas} \mathrm{al} \mathrm{referir} \mathrm{que} \mathrm{"...el} \mathrm{reino}$ de la necesidad y de la transitoriedad permanece anclado en las sombras de la esfera privada. Frente a ella se alza la publicidad,... como un reino de la libertad y la continuidad. A la luz de la publicidad todo se manifiesta tal como es, todo se hace a todos visible. " Cfr. Habermas, Jürgen, Historia y crítica de la Opinión Pública, GILI, Barcelona 2004, p.43 ${ }^{15} \mathrm{Cfr}$. Ibidem, p. 50

${ }^{16}$ Cfr. Garcia Llorente, Manuel, Ensayo sobre la vida privada, Editorial Encuentro, Madrid, 2011, p. 14
} 
Vida Privada e intimidad forman parte de ese espacio consagrado que le pertenece al individuo y que sólo con su consentimiento los terceros penetrarán hasta donde el mismo individuo permita. Llama la atención que la traducción al castellano del famoso trabajo de Warren y Brandeis The rigth to privacy ${ }^{17}$ sea el derecho a no ser molestado ${ }^{18} \mathrm{y}$ que dicho trabajo ya en la jurisprudencia de la Corte Suprema de los Estados Unidos sea entendido como el right to be alone es decir el derecho a estar solo. ${ }^{19}$ Es particularmente interesante ello pues en el uso cotidiano del lenguaje encontramos que privacy es usado como privado en tanto que en realidad privacy debe entenderse como íntimo.

Así cuando hablamos de intimidad nos estamos refiriendo a "aquello que está lo más dentro posible. No sólo lo que está en el interior del hombre sino lo que está el cogüelmo mismo de su interioridad humana" ${ }^{20}$ mientras que cuando nos referimos a vida privada hacemos alusión a un ámbito de mayor publicidad aunque igualmente contenida al consentimiento de la persona. "En cuanto la intimidad excede de una persona, aunque sea en un círculo cerrado como la familia, ya no es tal intimidad, sino vida privada"21 en ese sentido la característica esencial de la intimidad será su impermeabilidad. ${ }^{22}$

Así, aún cuando intimidad y vida privada se encuentran por decirlo coloquialmente, en la misma bolsa, obedecen a contenidos distintos y por tanto el tratamiento jurídico que se les puede dar también es diverso. Baste como ejemplo lo que sucede en materia de la llamada autodeterminación informativa y en concreto cuando se buscan proteger datos personales. Ahí, se hace la diferencia entre dos categorías de datos, aquellos denominado sensibles y los datos en general, atribuyendo a los primeros una vinculación con la vida íntima y a los segundos una vinculación con la vida privada trayendo como consecuencia inmediata que para hacer tratamiento de los primeros se requiere

\footnotetext{
${ }^{17}$ Cfr. Warren, S. D. Y Brandeis, L. D. El derecho a la intimidad, edición a cargo de Benigno Pendás y Pilar Baselga, Madrid, Civitas, 1995

${ }^{18}$ Sirva como ejemplo el magnífico libro asi titulado de CARRILLO, Marc, El Derecho a no ser molestado, Thomson Aranzadi, Navarra, 2003.

$19 \mathrm{lb}$ idem, p. 15. Al respecto el autor refiere que: "De forma más concreta, el derecho norteamericano la define como la potestad del titular a vivir sólo y a no ser molestado, que permite al individuo decidir soberanamente sobre su independencia personal" De igual manera lo refiere José María Desantes "...ya en 1902 la jurisprudencia americana definió la intimidad como el derecho a estar sólo...." Cfr. Desantes Guanter, José Maria, Derecho a la información, COSO, Valencia, 2004, p.230

${ }^{20} \mathrm{Cfr}$. Desantes Idem.

${ }^{21}$ Cfr. Ibidem, p. 233

${ }^{22} \mathrm{Cfr}$. Carrillo, op. cit. p. 51. Vale mucho la pena la definición que el autor construye de la intimidad al referir que es "...aquella que podríamos definir como el derecho de la persona no sólo a reservarse una esfera de vida propia y como tal impermeable a los demás, sino también a disponer de la capacidad de impedir o limitar la posterior difusión y eventual manipulación o instrumentalización de una información lesiva".
} 
un consentimiento expreso del individuo que los otorga mientras que, para los segundos sólo es necesario un consentimiento tácito. Ello evidencia en el plano de lo cotidiano los alcances de esta diferencia entre vida privada e íntima.

A diferencia de lo que ocurre con la intimidad en su carácter cerrado, la vida privada es una especie de círculo más ampliado, así ya lo refiere por ejemplo el Tribunal Europeo de Derechos Humanos en el caso Costelo-Roberts vs U.K. ${ }^{23}$ o bien como posición extrema el Juez Black de la Corte Suprema de los Estados Unidos al referir que“...el concepto de privacy es un concepto amplio, abstracto y ambiguo..." ${ }^{24}$ Lo cierto es que hablar de vida privada y no de intimidad nos permite hablar ya no de pensamientos, ya no de sentimientos sino de acciones o palabras que si bien es cierto no son públicas también es cierto no son íntimas.

La vida privada se desarrolla en privado y no en la intimidad. La vida privada no es pública pues en principio se encuentra contenida por lugares y personas que entienden la naturaleza de la acción o de la palabra como algo que ha sido compartido pero que les impide compartir. Sólo para ilustrar de manera pedagógica lo que hemos venido refiriendo José María Desantes refiere que "La esfera de la vida pública sería como la señal verde: paso libre a la información siguiendo la máxima romana publica publice tractanda sunt, privata private. La de la vida intima equivaldría a la luz roja: en ningún caso se puede penetrar en el interior de la persona contra su voluntad porque tiene derecho a reservárselo. La de la vida privada representaría el ámbar: en principio no es escrutable, -privata private- ni difundible cuando no trascienda de la privacidad; pero sí cuando trasciende a la vida pública". ${ }^{25}$

\section{b) Segmentación algorítmica, análisis predictivo y machine learning observando y aprendiendo de nuestra vida privada}

En el año 2010 José Luis Piñar Mañas adelantaba de manera sugerente una pregunta que cobra sentido en este trabajo, ¿Existe la privacidad? ${ }^{26}$ Esta pregunta,

\footnotetext{
${ }^{23} \mathrm{Cfr}$. Ruiz Miguel, Carlos. El derecho a la protección de la vida privada en la jurisprudencia del Tribunal Europeo de Derechos humanos. Cuadernos Civitas, Madrid, 1994, pp. 34

${ }^{24} \mathrm{Cfr}$. Suárez, op. cit. Es imperante decir que así también lo entienden otros tribunales como por ejemplo la Corte Interamericana de Derechos Humanos en el caso Atala riffo y niñas vs. chile de 24 de febrero de 2012 donde refiere la Corte, en el párrafo 162 que "...la vida privada es un concepto amplio que no es susceptible de definiciones exhaustivas...". Esto no significa que no haya tratado de precisar el concepto, por el contrario en diversos casos que trataremos más adelante ha tratado de aproximarse a diversos componentes de esta vida privada. Lo mismo ocurre con el Tribunal Europeo de Derechos Humanos.

${ }^{25}$ Cfr. Desantes, op. cit. p. 230

${ }^{26}$ AA.W., Protección de datos personales. Compendio de lecturas y legislación, H. Cámara de Diputados - IFAI- ITAM, TIRO CORTO EDITORES, México, 2010, p.15
} 
10 años después de ser lanzada se actualiza, de cara a lo que en ese tiempo ha ocurrido, en el enfrentamiento entre la vida privada y los adelantos tecnológicos. La necesidad imperante de interpretar y procesar datos provenientes de las esferas privadas e íntimas de las personas ha evolucionado en un imperativo con la explosión de las llamadas redes sociales. El flujo interminable de datos respecto al comportamiento de las personas en su vida cotidiana ha supuesto el nacimiento de toda una industria tecnológica que se alimenta de ellos y que analiza sistemáticamente su impacto en el comercio, en la política o en cualquier otro ámbito de la vida humana. Los algoritmos hoy, constituyen esa herramienta tecnológica que nos permite entender y aprender de los datos arrojados por todos. Utilizaremos entonces el término algoritmo, "para referirnos a una serie lógica de pasos para organizar y actuar sobre un conjunto de datos con el objetivo de lograr rápidamente un resultado". ${ }^{27}$ Es sin lugar a equívocos, la herramienta que nos permite como humanidad trabajar con grandes cantidades de datos para entenderlos, interpretarlos y poder tomar decisiones con ellos.

En ese tenor, es inevitable recordar el famoso caso del supermercado Walmart conocido como "pañales-cerveza" ${ }^{28}$ donde a partir del procesamiento adecuado de los datos se tuvo un incremento sustancial de venta de ambos productos. Este ejemplo produce, al menos desde la óptica comercial, una imperiosa necesidad de entender el comportamiento del mercado respecto a los bienes y servicios que se requieren. Invariablemente un adecuado entendimiento de los datos en el sector comercial tendrá mejores resultados de venta. ¿Qué debemos saber de las personas para poder incrementar las ventas? ¡Todo! Así lo hemos podido constatar desde el famoso caso de la red social Facebook respecto al estudio que hizo sobre comportamiento emocional en $2014 .{ }^{29} \mathrm{Hoy}$, desarrollos tecnológicos como el Feel Data ${ }^{30}$ revelan la importancia de cada dato que emana desde nuestra vida privada. Ya volveremos más adelante a este tema.

Pero hoy en día la utilización de los datos y la información que emana de las personas va más allá de un mero análisis de datos. La llamada inteligencia

\footnotetext{
${ }^{27} \mathrm{Cfr}$. Ortiz Freuler, J. Y Iglesias, C. Algorithms e Inteligencia Artificial en Latin America: Un Estudio de implementaciones por parte de Gobiernos en Argentina y Uruguay, World Wide Web Foundation, 2018, p. 8.

${ }^{28}$ Para mayor abundamiento del caso https://www.businessintelligence.info/dss/ejemplo-data-mining-panales-y-cerveza.html. Última consulta el 5 de febrero de 2020.

${ }^{29} \mathrm{Al}$ respecto se puede consultar:

https://www.bbc.com/mundo/noticias/2014/06/140630_tecnologia_facebook_experimento_emociones_criticas_ Iv. Ultima fecha de consulta: 6 de febrero de 2020.

${ }^{30}$ Entendiendo por Feel Data "una nueva definición para cuantificar la intensidad emocional de los consumidores y de esta manera generar un análisis deductivo sobre los pensamientos que se involucran en el momento de la compra". Disponible en: https://www.forbes.com.mx/feel-data-nueva-forma-de-descifrar-a-los-clientes/ Última fecha de consulta el 25 de enero de 2020.
} 
artificial a través de la tecnología de aprendizaje no sólo procesa los datos que podemos suministrar, sino que es capaz de aprender de ella. Así, por ejemplo, el proyecto lifenaut ${ }^{31}$ es quizás uno de los que reflejan mejor lo que referimos. Lifenaut "es un proyecto de investigación basado en la web que permite a cualquier persona crear una copia de seguridad digital de su mente y código genético". ${ }^{32}$ Dicho proyecto tiene como objetivo principal "...explorar la transferencia de la conciencia humana a las computadoras / robots y más allá”. ${ }^{33}$

En este caso la simple creación de una base de datos se volvería irrelevante por si misma lo importante es la creación del Mindlife que no es otra cosa que "una base de datos de reflexiones personales capturadas en video, imagen, audio y documentos... que se pueden guardar, buscar, descargar y compartir con amigos", ${ }^{34}$ a efecto de crear una mente digital que eventualmente pueda ser reproducida luego de la muerte y que interactúe de la misma manera que la persona viva. El proyecto recolecta también el código genético que eventualmente podría reproducir un cuerpo que sea cargado con el Mindlife. ${ }^{35}$

Esta recopilación de aprendizaje no sólo se encuentra en proyectos que quizás nos parecen lejanos. Hoy en día una buena parte de la humanidad utiliza Spotify, Amazon, Netflix o Waze sólo por mencionar algunas, aunque en realidad me atrevo a asegurar que la mayoría de las aplicaciones con las que convivimos a diario vienen acompañadas de algoritmos de aprendizaje que logran segmentar nuestras preferencias cotidianas. Es notorio que la comodidad que podemos encontrar en la segmentación de preferencias es profunda. Para que nos desgastaríamos eligiendo música en un mar de posibilidades si todos los días nos gusta oír el mismo tipo de canciones. La máquina aprende de nuestras preferencias y nos arroja la música que nos gusta. Lo mismo ocurre con las películas o con los libros. Lo mismo ocurre con las rutas que elegimos a diario o nuestras aplicaciones de ejercicio. Lo mismo ocurre con nuestros amigos y con nuestras preferencias políticas, religiosas o de consumo. Un mundo de análisis predictivo del comportamiento humano.

Claramente el sueño de Aaron Swartz sobre el derribo de las fronteras de 126 la información en su famoso documento "Manifiesto por la Guerrilla del acceso abierto" ${ }^{36}$ se cumple a cabalidad. La información per se debe, dice Swartz,

\footnotetext{
${ }^{31}$ https://www.lifenaut.com/

${ }^{32} \mathrm{Idem}$

${ }^{33} \mathrm{Idem}$

${ }^{34} \mathrm{Idem}$

${ }^{35} \mathrm{Idem}$

${ }^{36}$ Disponible en: https://endefensadelsl.org/guerrilla_del_acceso_abierto-binder.pdf. Última fecha de consulta el 29 de enero de 2020.
} 
ser libre. Es curioso que nada de lo descrito anteriormente se podría realizar si el muro de la vida privada se hubiera robustecido. En su lugar a diario encontramos millones de aceptaciones para el tratamiento de datos que revelan estados de nuestra vida privada con la única finalidad de gozar de aplicaciones que nos hagan más cómoda la vida pero que en realidad suponen un ejercicio profundo de penetración en nuestros pensamientos, sentimientos y reacciones más profundas.

Ante esta realidad ¿habrá forma de proteger en algo nuestra privacidad? Algunos proponen que "la inteligibilidad del algoritmo o modelo de IA es clave, tanto para entender su efectividad como para asegurar la legitimidad de su implementación... La inteligibilidad cobra particular relevancia cuando se trata de sistemas que definen o afectan la provisión de un servicio público, o que en cualquier modo afecten de manera sustancial los intereses, derechos o libertades de una persona" ${ }^{37}$ pues claramente se percibe que los efectos de una tecnología pueden llevar a consecuencias negativas por no decir lamentables. Es claro que proponer datos a la máquina para que ésta los interprete, procese o tome decisiones puede conducir a situaciones que inclusive los humanos no entendemos. Así surge de inmediato la necesidad de

“(...) reducir la opacidad. Al extremo de opacidad se le suele llamar “caja negra": quizás podamos observar los inputs y outputs, pero nunca llegamos a saber qué sucede entre estos dos polos. Los sistemas que operan como una caja negra eliminan la posibilidad de realizar un control descentralizado sobre la calidad de los outputs, y minan la confianza de las poblaciones afectadas (...)"

Toda vez que no se sabe al día de hoy porque la inteligencia artificial toma las decisiones con las que puede sesgar a un grupo o lesionar los derechos humanos de otro.

Todo ello ha ocasionado que surjan declaraciones internacionales como la de Toronto ${ }^{38}$ en la cual se obliga a los desarrolladores de software a promover la transparencia e igualdad en la creación y revisión de algoritmos para terminar con los problemas de racismo y discriminación evitando con ello los llamados sesgos implícitos. Pero ¿pueden los algoritmos evadirse de ello pensando por

\footnotetext{
${ }^{37}$ Cfr. ORTIZ, op.cit. p. 10

${ }^{38}$ Disponible en: https://www.accessnow.org/cms/assets/uploads/2018/08/The-Toronto-Declaration_ENG_08-2018. pdf, Última fecha de consulta el 5 de febrero de 2020
} 
ejemplo es las llamadas “cajas negras”? Así refiere el preámbulo de dicha declaración que:

Desde la vigilancia policial hasta los sistemas de bienestar, el discurso en línea y la atención médica, por nombrar algunos ejemplos, los sistemas que emplean tecnologías de aprendizaje automático pueden cambiar o reforzar enormemente y rápidamente las estructuras de poder o las desigualdades en una escala sin precedentes y con un daño significativo a los derechos humanos. Existe un conjunto sustancial y creciente de evidencia que muestra que los sistemas de aprendizaje automático, que pueden ser opacos e incluir procesos inexplicables, pueden contribuir fácilmente a prácticas discriminatorias o represivas si se adoptan sin las garantías necesarias ${ }^{39}$.

Claramente la preocupación sobre la opacidad y los daños que generan los llamados sistemas de aprendizaje son notorios y producen al día de hoy serias dudas sobre su oportunidad y aplicabilidad por la constante producción de efectos discriminatorios o represivas. Pero entonces ¿Es posible negarse a ser incluido en estos procesos? ¿Constituye un derecho humano exigir la garantía de la no inclusión en dichos procesos? ¿El derecho a la autodeterminación informativa ampara la libre elección de no ser segmentado?

\section{4. ¿Es posible una vida libre de algoritmos, segmentación 0 análisis predictivo?}

Las respuestas a las preguntas con las que cerramos el apartado anterior podrían partir de lanzar otra pregunta que es si toda la utilización de algoritmos para el procesamiento de grandes cantidades de datos por sí mismo genera una violación a nuestros derechos humanos y en concreto a nuestra vida privada o vida íntima. Tal parece que el procesar datos anónimos, desasociados de las

128 personas, sólo produce efectos positivos para entender comportamientos colectivos llevándonos a suponer en principio, un actuar sin menoscabo de la vida privada. En ese sentido claramente el tratamiento de Big Data ${ }^{40}$ estaría

\footnotetext{
${ }^{39}$ Idem

40 "El término datos masivos proviene originalmente del ámbito de las ciencias de la computación, y se refiere a un conjunto de datos cuyo tamaño excede al que puede manejar el software y hardware estándares disponible para capturar, almacenar y analizarlos". En Rodríguez, P., Palomino N., Moncada, J. El uso de datos masivos y sus técnicas analíticas para el diseño e implementación de políticas públicas en Latinoamérica y el Caribe, BID, Chile, 2017, p. 2
} 
destinado a ser una herramienta muy útil para la toma de decisiones. Es innegable destacar el aporte que en diversas materias se ha logrado a partir del tratamiento de grandes cantidades de datos para la toma de decisiones públicas tales como pobreza, crimen y seguridad, movilidad, transporte y salud ${ }^{41}$, las cuales sin la utilización de dichos datos jamás se hubieran podido resolver problemas cruciales para la vida cotidiana de las personas.

En el caso particular de los datos masivos el problema no radica en la captación, uso o difusión de los mismos sino en los sesgos que las nuevas tecnologías de aprendizaje pueden llevar a cabo en una toma de decisiones no supervisada y regulada por la mano humana. En ese sentido tendríamos necesariamente que distinguir entre:
a) Análisis descriptivo
b) Análisis predictivo
c) Análisis prescriptivo

Claramente el análisis descriptivo de datos lo podemos considerar libre de causar de manera autónoma una violación a los derechos humanos. La llamada analítica descriptiva consistirá en el almacenamiento y agregaciones de datos históricos, permitiéndonos observar y visualizar como ha funcionado una empresa o una política pública. ${ }^{42}$

En donde podemos comenzar a observar algún tipo de impacto será en el llamado análisis predictivo.

(Éste) se construye sobre la analítica descriptiva y usa modelos estadísticos avanzados para añadir a nuestra base de información datos que no conocemos. Esto se traduce en técnicas como la predicción de valores futuros en series históricas de precios y demandas, pero también la clasificación automática de textos u operaciones, o la segmentación de clientes." ${ }^{43}$

La presencia de segmentación o de sesgos para tomar decisiones puede llevar a suponer segregaciones o profundos impactos en la vida privada de las personas respecto a su libertad personal y toma de decisiones.

\footnotetext{
${ }^{41}$ Idem, p. 3

${ }^{42}$ Sobre el particular consultar https://www.iic.uam.es/big-data/analitica-descriptiva/. Última fecha de consulta el 12 de febrero de 2020.

${ }^{43} \mathrm{Idem}$
} 
La problemática con la analítica predictiva radicará en la carencia del ser humano para impedir el tratamiento de una información que lo encasille, arrincone o lo clasifique respecto a un producto, servicio determinado o porque no, respecto a una preferencia política concreta. Así, por ejemplo, las nuevas generaciones de refrigeradores que pueden entender nuestro comportamiento de almacenaje y consumo de leche a la semana provocarán que el supermercado nos abastezca la misma leche y en la misma cantidad por toda nuestra vida sin que tengamos posibilidad de intervenir en la decisión. Lo anterior no es nuevo ya que sucede, como lo relatábamos en líneas anteriores, en prácticamente toda la nueva tecnología que acompaña a nuestros teléfonos móviles. Spotify predice, a partir del comportamiento, qué tipo de canciones queremos oír y nos presenta nuestra lista de posibles preferencias a partir de su lectura de nuestro comportamiento. La predicción está llamada a suprimir la elección humana por razones comerciales o por razones políticas, pero también por razones de bienestar o comodidad pues la alimentación de datos de esa segmentación la otorgamos cada uno de nosotros.

El tercero de los grupos respecto al manejo de datos es el llamado análisis prescriptivo.

Supone el nivel más alto de analítica y explota los niveles anteriores junto con estrategias de optimización operativa para indicarnos qué acciones de negocio proporcionarán los mejores resultados. Mediante la analítica prescriptiva podemos obtener recomendaciones automatizadas sobre el momento idóneo para ejecutar pedidos, mantenimientos u otras operaciones cuantificables del negocio. Con la analítica prescriptiva podemos saber qué debemos hacer para optimizar nuestro negocio. ${ }^{44}$

Como podemos observar en este nivel tenemos un riesgo serio de posibles violaciones a derechos humanos, pues en la automatización de la respuesta no hay intervención humana. El procesamiento de datos que inicia con su captación estará orientado fundamentalmente para la creación de respuestas automatizadas que resuelvan problemas. Un caso que puede evidenciar los alcances de esta automatización de la respuesta es sin duda el caso del software Pre$d P o l$ en Uruguay el cual estaba destinado a conseguir una mejor toma de decisiones en materia de presencia policiaca en las llamadas zonas de "alto riesgo”. Los resultados vinieron acompañados de preguntas interesantes sobre la 
automatización de la presencia de policías en algunas zonas que el algoritmo entendió como zonas de riesgo y que en ocasiones no lo fueron. La máquina, de manera automática, generó una discriminación sobre dichas zonas. Los resultados fueron escasos y sólo evidenció los sesgos clasistas y racistas. ${ }^{45}$ En ese sentido cabría destacar que:

Un informe publicado en 2016 por investigadores del Human Rights Data Analysis Group (HRDAG) sugiere que este tipo de programas generan un circuito de retroalimentación: Ante más presencia policial, más probabilidad de detectar un ilícito. Cuantos más ilícitos registrados en un área, más probabilidad de que se envíen fuerzas policiales. De acuerdo a HRDAG esto lleva a que los agentes sean enviados repetidamente a las mismas zonas de la ciudad, generalmente aquellas donde se concentran minorías raciales, independientemente de la verdadera tasa de criminalidad en esa área ${ }^{46}$

Ante este panorama predictivo - prescriptivo vale la pena preguntarnos si el derecho a la autodeterminación informativa no ampara el derecho para que cualquier persona pueda evadirse de estos tratamientos de datos. En principio la solución es muy sencilla pues aquel derecho contempla en sí mismo la capacidad que tenemos de entregar o no datos a un tercero. De igual manera contempla la posibilidad de eliminar los datos de todo aquel que los tenga o bien de oponernos a los determinados tratamientos de datos. De manera eficiente, cobraría sentido afirmar, que la autodeterminación informativa supone de inicio el derecho a evadirme de procesos automatizados de datos personales.

En consecuencia de lo anterior y siguiendo el principio pro homine que se ha acuñado a nivel internacional como bandera matriz de la defensa de los Derechos humanos ${ }^{47}$ tendríamos necesariamente que conminar al Estado

\footnotetext{
${ }^{45}$ Cfr. Ortiz, op. cit. pp. 27-30

${ }^{46}$ Idem.

${ }^{47}$ Podemos entender este principio como: "criterio hermenéutico, criterio interpretativo prevalente en materia de derechos humanos, guía interpretativa, conjunto de parámetros-guía, conjunto de directrices para la interpretación y aplicación del derecho de los derechos humanos, norma subyacente, norma "no enunciada"), regla de preferencia-predominio, regla primaria no escrita inherente al derecho internacional de los derechos humanos, principio ordenador, vector que da sentido y jerarquiza al sistema normativo, principio general del derecho de los derechos humanos, polo cristalizador del orden público en materia de derechos humanos, norma de justicia objetiva, canon catalizador de los objetivos y fines de todo el sistema de protección de los derechos humanos, principio con arreglo al necesario derecho de la societas gentium, regla que está en la cúspide del complejo corpus iuris de los derechos humanos que prioriza a la persona humana frente a otros sujetos internacionales, punto de apoyo de la formación de un ius commune transnacional, regla amalgamadora del derecho interno e internacional de los derechos huma-
} 
a otorgar una garantía de protección a todas aquellas personas que buscaran evadirse de estos tratamientos de datos al tratarse de una intromisión directa a su vida privada. Es decir que en materia de interpretación constitucional y convencional del derecho a la autodeterminación informativa el Estado tendría que proteger a todo individuo que buscara evadirse de los tratamientos de segmentación, predicción o prescripción algorítmica y establecer todos los mecanismos necesarios para limitar el mismo.

Llevando al extremo la práctica que se realiza en algunas partes del mundo sobre el llamado Derecho al olvido digital, éste se encontraría encaminado a una eliminación por completa de los datos personales que se han entregado a diversos responsables y que asociados con el nombre $u$ otros datos que nos identifican, deben ser eliminados de cualquier plataforma digital empezando con los buscadores. Este derecho al olvido digital manifiesta una vertiente de evadirnos del tratamiento de datos personales que en determinadas condiciones podríamos llevar al tratamiento automatizado de los mismos cuando generan patrones de identificación de las personas. En ese sentido en términos del Reglamento Europeo de Protección de Datos Personales, ${ }^{48}$ este derecho al olvido es una mera manifestación del derecho de supresión, pero vinculado a los buscadores de internet que tiene por objeto protegernos de la lesión que implica en nuestra vida privada la difusión de información en aquellas plataformas.

Ante ello cabe preguntarnos si ¿podríamos construir en el derecho al olvido digital una vertiente que impacte directamente en la supresión de datos que manejen algoritmos de predicción y prescripción? Sabemos que las herramientas jurídicas que se han construido (derecho a la vida privada, derecho a la autodeterminación informativa y derecho al olvido digital en colaboración con el principio pro homine) nos arrojan una posible salida para elaborar la posibilidad de evadirnos de este tipo de tratamientos. Los pasos a considerar serían: a) no consintiendo en el tratamiento de los datos, b) revocando nuestro consentimiento si ya los hemos dado, c) solicitando la cancelación o supresión de los datos, d) solicitando la eliminación y olvido de nuestra presencia digital en cualquier plataforma digital que trate datos de manera automatizada.

Por último, habría que preguntarnos si ello es una utopía. Habría que contestar esa pregunta con otras. ¿La defensa de los derechos humanos lo es? ¿Por el desarrollo tecnológico estamos obligados a ceder nuestros derechos? ¿Vale

nos, base de un nuevo ius Gentium" Cfr. Drnas De Cément, Z., La complejidad del principio pro homine, CIDH, Buenos Aires, 2015, Disponible en: http://www.corteidh.or.cr/tablas/r33496.pdf. Última consulta el 10 de febrero de 2020. ${ }^{48}$ Disponible en: https://eur-lex.europa.eu/legal-content/ES/TXT/?uri=celex\%3A32016R0679. Última consulta el 19 de febrero de 2020. 
más un algoritmo que predice la conducta que la defensa de la vida privada? En la respuesta que demos a estas interrogantes encontraremos el núcleo central del derecho a una vida de algoritmos.

\section{Conclusiones}

El profundo y vertiginoso cambio tecnológico ha venido acompañado de una transformación clara de los alcances y defensa de la vida privada. Así, la nueva tecnología presenta desafíos que el derecho debe atender sin el menoscabo de las ventajas y beneficios que dichos adelantos suponen.

El efecto de la inteligencia artificial a través de la llamada machine learning, que logra generar un análisis de datos de manera predictiva o de manera prescriptiva, están suponiendo un estupendo semillero de problemas jurídicos vinculados no sólo con la vida privada sino también con el menoscabo de la libertad o con la generación de situación de desigualdad en prácticamente todo el mundo. La ilusión de comodidad y bienestar se desdibuja a partir de la invasión a la privacidad, discriminación y segmentación.

La autodeterminación informativa como un derecho derivado de la vida privada supone el control y consentimiento del tratamiento de datos personales teniendo como alcance claro y definido la posibilidad de decidir sobre a quién entregamos nuestros datos, cómo queremos que sean tratados y en su caso la eliminación de dichos datos.

Los alcances de esta autodeterminación informativa proponen desdoblarse a un nuevo derecho que el Estado debería garantizar, un derecho que permita a la persona aislarse de tratamientos automatizados de datos predictivos 0 prescriptivos sin menoscabo de seguir obteniendo servicios de diversa índole. El Estado deberá reconocer la injerencia de los algoritmos de segmentación predictiva o prescriptiva en la vida privada de las personas, en el derecho de igualdad o en la libertad para la toma de decisiones y establecer mecanismos suficientes de protección.

\section{Bibliografía}

Arendt Hannah, La condición humana, PAIDOS, Barcelona, 2005

AA.VV., Protección de datos personales. Compendio de lecturas y legislación, H. Cámara de Diputados - IFAI- ITAM, TIRO CORTO EDITORES, México, 2010.

Beitz, Charles, La idea de derechos humanos, España, Marcial Pons, 2014.

Campuzano Tome, Herminia, Vida Privada y Datos Personales, Madrid, Tecnos 2000. 
Carbonell, Miguel, El ABC de los derechos humanos y el control de convencionalidad, México, Porrúa, 2014.

Carrillo, Marc, El Derecho a no ser molestado, Thomson Aranzadi, Navarra, 2003.

Castán Tobeñas, José, Los derechos del hombre, Madrid, Reus, 1992.

Desantes Guanter, José María, Derecho a la información, COSO, Valencia, 2004.

Drnas De Cément, Z., La complejidad del principio pro homine, CIDH, Buenos Aires, 2015

García Llorente, Manuel, Ensayo sobre la vida privada, Editorial Encuentro, Madrid, 2011. Habermas, Jürgen, Historia y crítica de la Opinión Pública, GILI, Barcelona 2004.

Harari, Yuval, Homo Deus, Debate, México, 2016.

Hierro, Liborio, Los derechos humanos, una concepción de la justicia, España, Marcial Pons, 2016.

Lucena-Cid, Isabel, El impacto de las nuevas tecnologías en los derechos humanos, In: Revista Aporia Jurídica (on-line). Revista Jurídica do Curso de Direito da Faculdade CESCAGE. 8a. Edição. Vol. 1 (jul/dez-2017).

Molnar, Petra, LEX, Gill, Bots at the gate: A human rights analysis of automated decisión - making in Canada s inmigration and refugee system, Citizen Lab University of Toronto, 2018. Disponible en: https://citizenlab.ca/wp-content/ uploads/2018/09/IHRP-Automated-Systems-Report-Web-V2.pdf, última fecha de consulta el 10 de febrero de 2020.

Morales Vega, Luisa Gabriela, Derechos humanos y su interpretación en la Corte, México, DOFISCAL, 2018.

Ortiz Freuler, J. Y Iglesias, C. Algorithms e Inteligencia Artificial en Latin America: Un Estudio de implementaciones por parte de Gobiernos en Argentina y Uruguay, World Wide Web Foundation, 2018.

Pérez Luño, Antonio E, Los derechos fundamentales, Madrid, Tecnos, 2004.

Ramirez García, H. Pallares Yabur, P. Derechos humanos, Oxford, México, 2011

Rheingold, Howard, Multitudes inteligentes. La próxima revolución social, Barcelona: Gedisa, 2004.

Rodríguez, P., Palomino N., Moncada, J. El uso de datos masivos y sus técnicas analíticas para el diseño e implementación de políticas públicas en Latinoamérica y el Caribe, BID, Chile, 2017.

Ruiz Miguel, Carlos. El derecho a la protección de la vida privada en la jurisprudencia del Tribunal Europeo de Derechos humanos. Cuadernos Civitas, Madrid, 1994.

Stallman, Richard, A radical proposal to keep your personal data safe, The Guardián, Abril 2018. Disponible en: https://www.theguardian.com/commentisfree/2018/ apr/03/facebook-abusing-data-law-privacy-big-tech-surveillance. Fecha de última consulta el 6 de febrero de 2020.

Suarez Crothers, Christian. El concepto de derecho a la vida privada en el derecho anglosajón y europeo. Rev. derecho (Valdivia). [online]. dic. 2000, vol.11 [citado 24 Noviembre 2015]. Disponible en la World Wide Web:

< http://mingaonline.uach.cl/scielo.php?script=sci_arttext t pi$\mathrm{d}=$ S071809502000000100010\&lng=es\&nrm=iso > 
Warren, S. D. Y Brandeis, L. D. El derecho a la intimidad, edición a cargo de Benigno Pendás y Pilar Baselga, Madrid, Civitas, 1995

Recursos Web

https://www.businessintelligence.info/dss/ejemplo-data-mining-panales-y-cerveza. html

https://www.bbc.com/mundo/noticias/2014/06/140630_tecnologia_facebook_experimento_emociones_criticas_lv.

https://www.forbes.com.mx/feel-data-nueva-forma-de-descifrar-a-los-clientes/ https://www.lifenaut.com/ https://endefensadelsl.org/guerrilla_del_acceso_abierto-binder.pdf https://www.accessnow.org/cms/assets/uploads/2018/08/The-Toronto-Declaration ENG_08-2018.pdf.

https://www.iic.uam.es/big-data/analitica-descriptiva/ http://www.corteidh.or.cr/tablas/r33496.pdf https://eur-lex.europa.eu/legal-content/ES/TXT/?uri=celex\%3A32016R0679 